INBRIEF

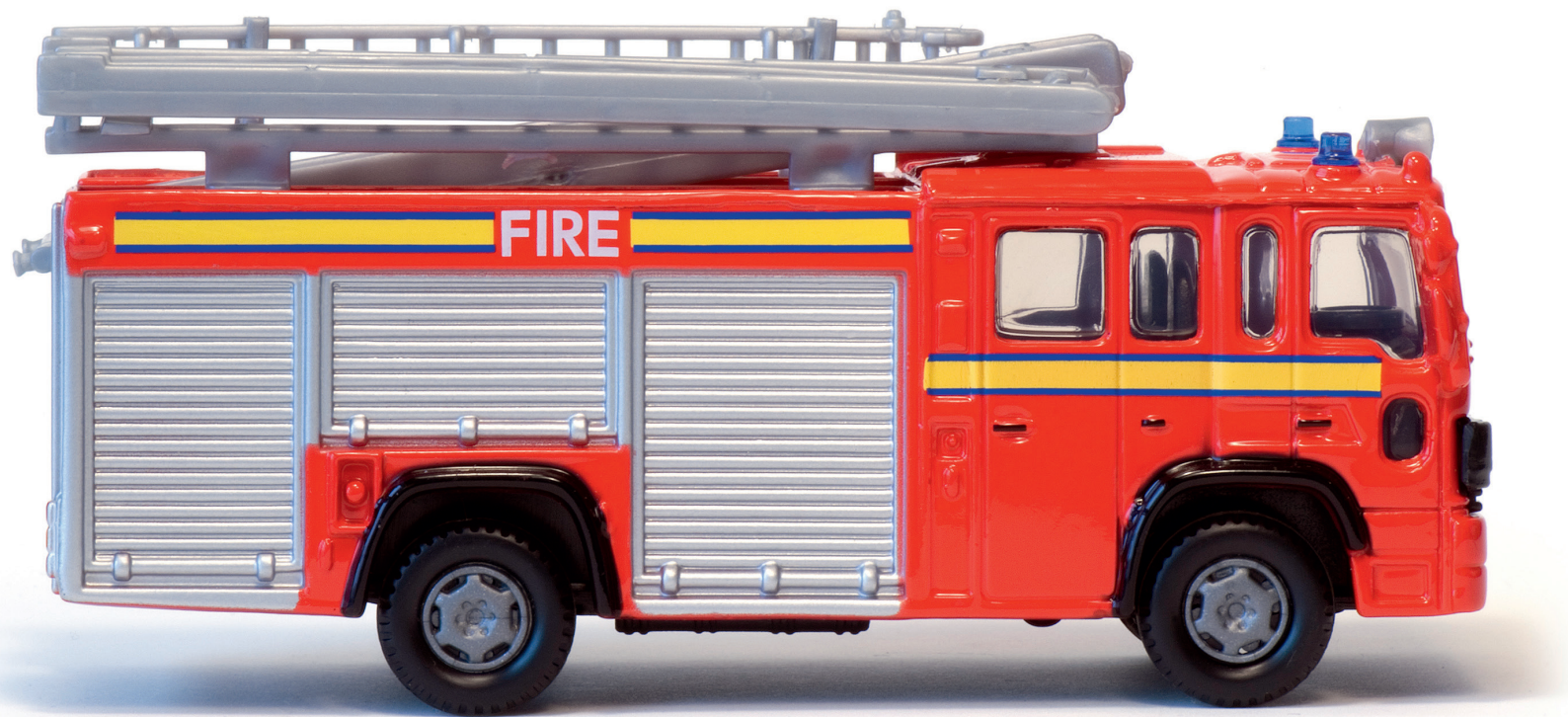

VISUALISATION

\title{
London's burning: Assessing the impact of the 2014 fire station closures
}

\author{
What effect have fire station closures had on response times in central London? Benjamin M. Taylor investigates
}

$n$ the face of ongoing austerity measures imposed by the British government, the consequences of cuts to public services are beginning to unfold. One of the most contentious decisions taken in 2014 related to the closure of 10 fire stations and the loss of 552 fire-fighters around the central London area. These measures were a result of the London Fire Brigade (LFB) being tasked with making savings of almost £29 million.

The affected stations were Belsize, Bow, Clerkenwell, Downham, Kingsland, Knightsbridge, Silvertown, Southwark, Westminster and Woolwich. These stations were selected for closure (presumably) so as to minimise any disruption to coverage and service in affected areas. Indeed, the LFB said at the time that services would not be impacted. The Fire Brigades Union (FBU), however, had a different perspective. Paul Embery, regional secretary of the FBU, said: "It will be only a matter of time before someone dies because a fire engine did not get to them in time" (bbc.in/1reZEb7).
To its credit, the LFB collects information on all call-outs - including the type of fire, date, time of day, spatial location and response time - which allows researchers to analyse the impact that station closures have had. In fact, in preparation for the closures, the Open Data Institute used this information to work out that average response times across London were likely to increase only marginally, from $5 \mathrm{~min} 34 \mathrm{~s}$ to $5 \mathrm{~min}$ $37 \mathrm{~s}$. However, in parts of the city directly affected by the station closures, the effect was expected to be more pronounced (london-fire.labs.theodi.org/).

Now, two years on, I have sought to bring some statistical rigour to these efforts and to map the response times at a more granular level, using data on dwelling fires only - as this type of fire accounts for over $80 \%$ of fire-related fatalities. Across the UK, there were 39600 dwelling fires in 2013-2014 (bit.ly/1rf6yNu).

\section{Areas at risk}

London is divided into 33 districts -32 boroughs plus the City of London. The

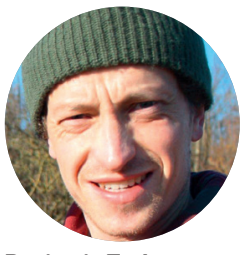

Benjamin Taylor completed his $\mathrm{PhD}$, "Sequential Methodology with Applications in Sports Rating" at Lancaster University in 2010. He joined the CHICAS research group, headed by Professor Peter Diggle, as a research associate in 2010 and subsequently as a lecturer in biostatistics in 2013.

This article is based on Benjamin Taylor's paper, "Spatial Modelling of Emergency Service Response Times", which is published in the Journal of the Royal Statistical Society, Series A at bit.ly/1TBkmLA boroughs can be divided further into wards; however, my analysis sought to understand the effect of the station closures at the sub-ward level.

Deciding which spatial areas may be at risk of increased response times following the closures is not just a question of producing maps of the raw data. The time an engine takes to respond to a call depends at what time of day and on which day of the week the fire occurs, as road traffic and staffing levels will vary accordingly. Response times also depend on the level of service provision in the vicinity - that is, the number of fire stations able to respond in a reasonable time to a call - and the demands on those stations being placed by other calls at the time of the fire, the effect of which might be that an engine has to be deployed from a station further out. There will, of course, be other influencing factors not measured by the available covariates.

The main aims of my analysis were to identify spatial regions that had potentially been affected by the fire 


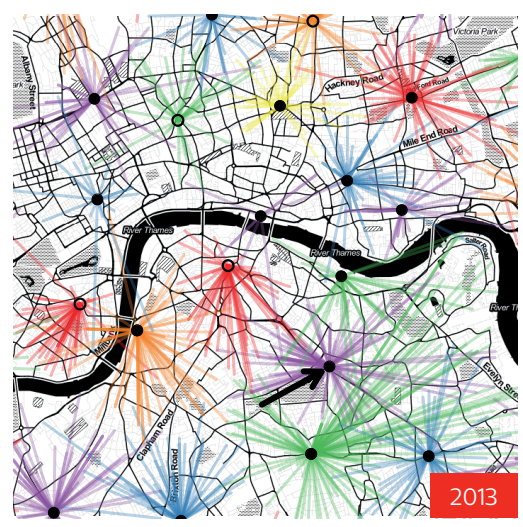

station closures and to determine if response times had changed in those areas. Response times are partially governed by the redeployment strategy adopted by the LFB which is constantly evolving, as can be seen in Figure 1.

Specifically, I looked for areas in which the response times (accounting for time of day, a weekend effect and the level of local service provision) seemed higher in 2014 and 2015 compared with 2013 and 2012.

Figure 2 gives an idea of the size and location of regions potentially affected by the closures and how these have changed between 2014 and 2015. For residents living in the affected areas, the consequences of the closures could be serious. Following the closures, the LFB has only been able to respond to around $50 \%$ of calls in some of these regions (marked as red, blue or green) within its 6-minute target. Before the closures, there was no problem with response times at all.

\section{Fire alarm}

In my analysis, I assume a proportional hazards spatial survival model for the response times. While there may be other sensible modelling alternatives and methods for identifying potentially affected regions, my analyses indicate that the 2014 closures have had a negative impact on response times in the central London area.

To find out the substantive implications of these closures - that is, whether increased response times have led to more injuries and deaths, as the FBU suggested it would - it
FIGURE 1 Maps of dwelling fire locations and deploying stations connected by coloured lines in 2013, 2014 and 2015. Different colours are used to help distinguish the responses from each station. Solid markers are operational fire stations, hollow markers are the locations of the 10 fire stations closed in 2014. These plots show how the London Fire Brigade has adjusted its response strategy in light of the closures; note that the strategy employed in 2015 is different from that employed in 2014. For example, consider Old Kent Road station, highlighted by an arrow. In 2014 this station was responding mainly to fires to the east, but in 2015 responded in all directions, as it did in 2013

FIGURE 2 Areas where response times were higher in 2014, 2015, and both 2014 and 2015 than they were in 2012-2013, adjusting for time of day, a weekend effect and level of service provision. The bold line marks a $4 \mathrm{~km}$ buffer around the convex hull of the closed fire stations; we only consider differences in response times within this region in the present article. The results are presented on a $500 \mathrm{~m}$ by $500 \mathrm{~m}$ square grid of small areas
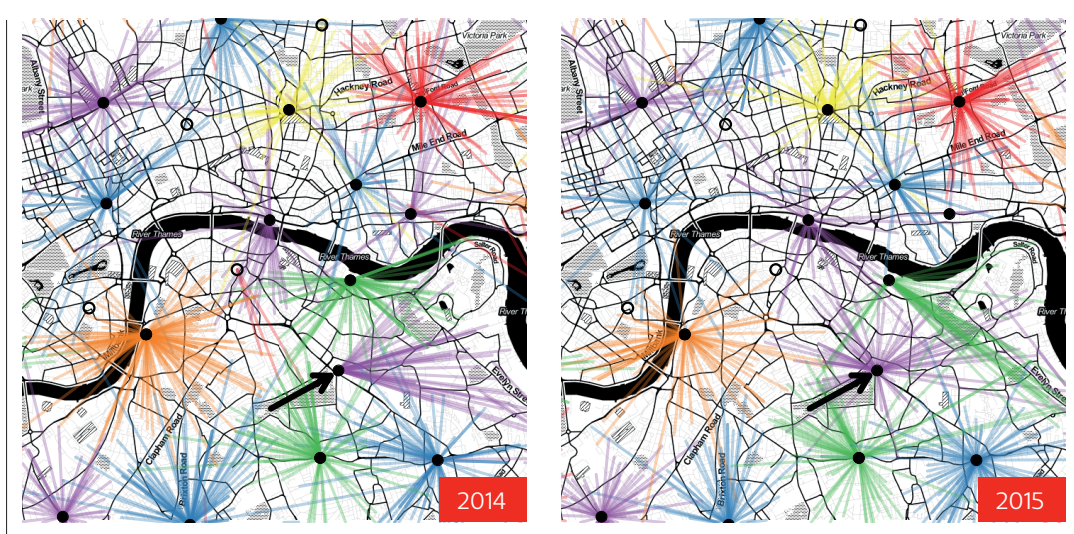

is necessary to link response time data to data from the ambulance service and from hospitals to follow up health outcomes as a result of fires, comparing the health outcomes of victims in 2012-2013 to those in 2014-2015. Changes in the cost of damage to property resulting from fires could also be compared if the right data were available.

Two years ago, the LFB declared publicly that services would not be affected by station closures. Two years on, the impact of the closures would seem to be more substantial than anticipated.

\section{Acknowledgements}

Map tiles by Stamen Design, under CC BY 3.o. Data by OpenStreetMap, under ODbL. The London Fire Brigade data are subject to the Open Government Licence: http://www.nationalarchives. gov.uk/doc/open-government-licence/.

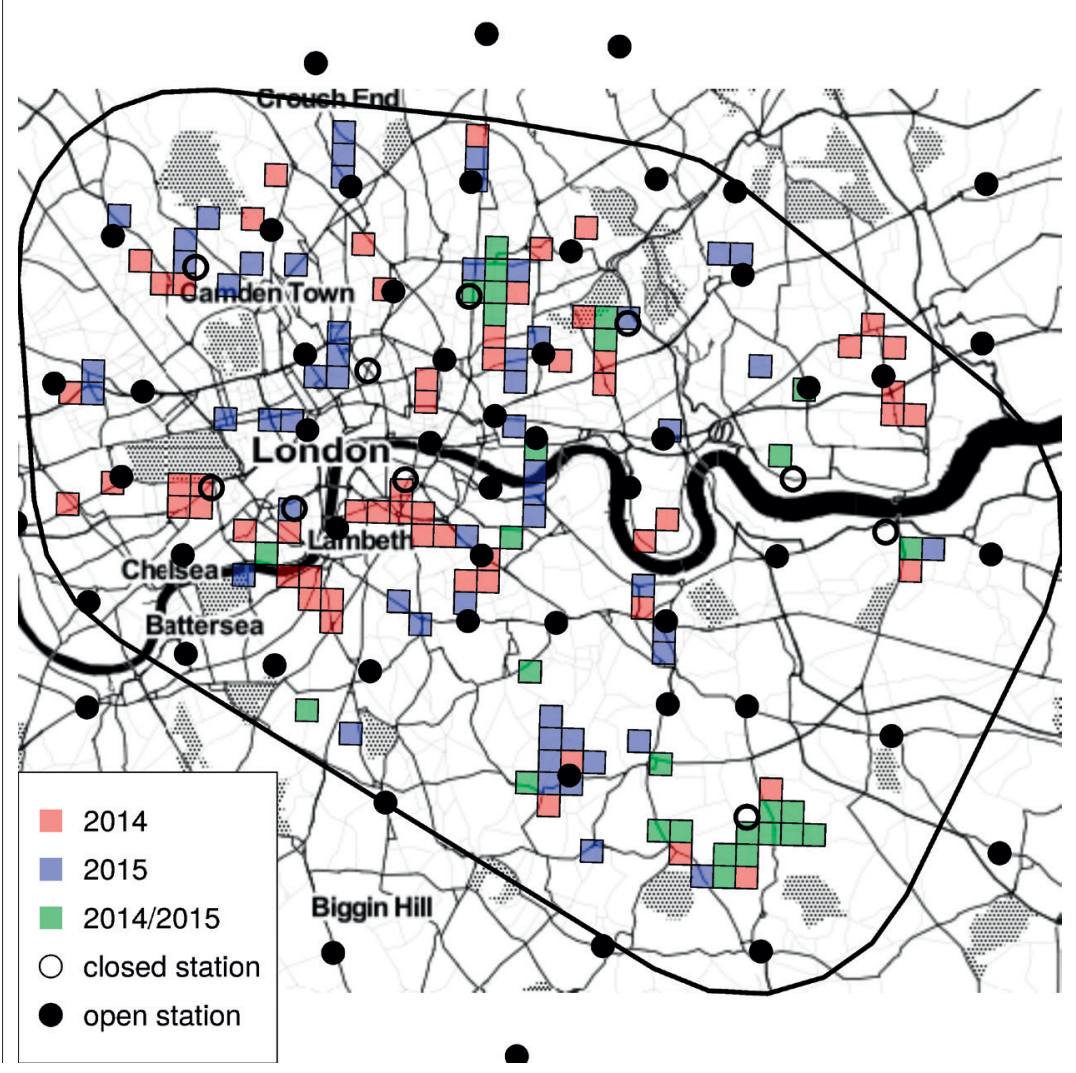

June 2016 | significancemagazine.com | 11 